\title{
WCC 2017-C-103: EMERGENCY SURGICAL RETRIEVAL OF EMBOLIZED ATRIAL SEPTAL DEFECT CLOSURE DEVICE FROM LEFT ATRIUM
}

\author{
Praveen Dontineni, Ramesh Chandra Mishra, Amaresh Rao Malempati
}

\begin{abstract}
:
With the introduction of technological improvements, invasive cardiologic interventions have become increasingly used alternatives to surgery. Despite its numerous benefits, serious and potentially lifethreatening complications of invasive cardiology interventions may occur. Atrial septal defect is usually closed in the cardiac catheterisation laboratory using atrial septal occluder (ASO) device. One of the complications associated with the procedure is dislodgement of the device. We present a case of percutaneous trans catheter closure of an ostium secundum atrial septal defect in a 21 years old female, complicated by device dislodgement to the left atrium necessitating emergency surgical retrieval.

Keywords: Atrial septal defect (ASD), occluder devices, Embolization.
\end{abstract}

\section{INTRODUCTION:}

Atrial septal defect (ASD) is one of the most common congenital heart disease (3.78 per 10000 live births.) For several decades' surgical closure has been considered the standard method of repairing a secundum ASD. Surgical treatment is safe and effective but the complications relate to thoracotomy, bleeding, arrhythmia, post-pericardiectomy syndrome [1]. There have been some creative efforts by interventional cardiologists in closing ASD using various devices. ASD closure device was first described by Ring et al. in 1976.Percutaneous ASD closure has become an

Article received on 25 FEB 2017, published on 08 MAR 2017. Praveen Dontineni1, Ramesh Chandra Mishra2, Amaresh Rao Malempati3

${ }^{1}$ Senior Resident, Department of CT Surgery, NIMS, India

${ }^{2}$ Professor, Department of CT Surgery, NIMS,India.

${ }^{3}$ Addl. Professor, Department of CT Surgery, NIMS,India.

Corresponding author: Praveen Dontineni

Email: doctorpraveendontineni@gmail.com increasingly simplified procedure over the past decade. Complications commonly associated with ASD closure device included residual shunt, device malposition or embolization, thrombosis over the vena cava or atrium, erosion and perforation of the heart, and infective endocarditis. Device embolization or erosion could result in catastrophic complications such as left ventricle outflow tract obstruction, cardiac rupture or cardiac tamponade. Chun reported a case with aorta-to-right atrial fistula three months after device implantation [2]. The use of dedicated devices for ASD closure by experienced operators results in low rates of device dislodgement, migration or embolization and improves sealing performance. A cause of failure is early embolization. These have resulted in varying symptom and hemodynamic compromise, most requiring surgical retrieval [3]. We report a case of ASD closure device embolization to left atrium.

\section{CASE REPORT:}

A 21-year-old female presented with symptoms of breathlessness of functional class 2 since the past 6 months and underwent echocardiography, which showed a $19 \mathrm{~mm}$ ostium secundum ASD having well defined rim with left to right shunt and LVEF of 60 $\%$ and RVSP of $35 \mathrm{~mm} \mathrm{Hg}$. The patient therefore seemed to be a good candidate for ASD closure using $22 \mathrm{~mm}$ Amplatzer Septal Occluder (ASO). Standard practice was adopted for deployment of the device successfully. On post procedure day 2, routine trans thoracic echo showed the device in left atrium stuck to interatrial septum. Although the patient was hemodynamically stable, urgent surgical intervention was recommended to retrieve the device and close the defect. The patient was taken emergently to the operating theatre, median sternotomy was done and cardiopulmonary bypass was established by aortic and bicavalcannulation. The aorta was clamped and heart was arrested with antegrade cardioplegia. The right atrium was opened and a large secundum ASD was found. The device was covered with recent red thrombus and was found to be 
embolized partially into the left atrium, lying just below the inferior margin of the ASD defect. Through the ASD, the device was retrieved and the defect was closed with glutaraldehyde treated autologous pericardial patch. Patient was gradually weaned from cardiopulmonary bypass. Patient was extubated after 4 hours of mechanical ventilation. The patient had an uneventful recovery and was discharged on post op day five.

\section{DISCUSSION:}

Trans catheter occlusion techniques have become an increasingly used alternative to surgical closure in selected cases of the ASD and various devices are available for this [3]. Most secundum ASDs can be closed by Amplatzer device. Common criteria for a patient being suitable for closure by Amplatzer include a defect with less than or equal to $34 \mathrm{~mm}$, a $4-\mathrm{mm}$ rim of septal tissue surrounding the defect and sufficient distances from surrounding valves and structures. The hazards of ASO are thick profile of device and high amount of nitinol in device and the potential for nickel toxicity. ${ }^{1}$ Trans catheter closure of ASD has comparable results to surgical closure [3]. Absence of residual shunts and late thrombo embolic events are in favor of surgical closure of ASD. Minimally invasive techniques address cosmetic angle without compromising results. The need for lifelong antiplatelet agents and SBE prophylaxis must be weighed against the disadvantage of a small incision. A promising early result by device closure does not guarantee a favorable late outcome [4]. Embolization does not commonly occur but if develops, the reasons are insufficient rim around the defect, early release of device, and mismatch between the size of ASD and Amplatzer which is due to the use of small Amplatzer for a large ASD. Transesophageal echocardiography (TEE) performed during Amplatzer placement seems to be a potential contributor to prevent technical errors and occurrence of complications [1]. Trans catheter devices can be associated with failures and most importantly, with life threatening complications. The most important reason for acute failure of these devices is poor patient selection, device selection, or both [5]. A part or the entire device might embolize to the right or left atrium, main pulmonary artery, or even to other parts of the vascular tree on both the right and left sides of the circulation. Once the device is detached from its cable, it becomes difficult to retrieve, and depending on its location, it might even become lethal [5]. Hui Chung Wu et al. (2013) mentioned in their study that between January 2000 and December 2010, a total of 508 patients underwent closure of secundum type ASD using an Amplatzer septal occluder (ASO). Six of the patients $(1.2 \%)$ had device embolization or migration warranting surgical retrieval and repair [2]. In a report by David et al. 186 patients had atrial defects closed by means of Amplatzer device with no major complications. There were eight $(4.3 \%)$ minor complications including four device embolization with two removed percutaneously and two surgically. Also, four instances of arrhythmia comprising three transient, and one persistent complete heart block [1]. Percutaneous retrieval of the embolized device is possible in about $70 \%$ of cases. However, some authors suggest that embolization of device is always an indication for emergency surgical retrieval, which also permits a direct inspection of intra-cardiac structures that may have become injured [6]. In our patient, we also preferred surgical approach due to close relation of device with mitral valve and the left ventricle. In conclusion, a careful echo cardiographic assessment and procedure planning should be done for a percutaneous intervention. In addition, surgical back-up must be available in the hospital to cope with potentially lethal acute complications. Although closure of left to right shunting defects by percutaneous occluder devices has a lot of advantages, device embolization is still a major complication. Surgical management is the only method to remove embolized devices after failed percutaneous retrieval attempts. In this circumstance, surgical back-up and urgent management strategies should be well planned.

\section{REFERENCES:}

1. Tabaee AS, Rostami AR, Ghafari R. Surgical removal of an embolized amplatzer device from left ventricle. Iranian Cardiovascular Research Journal 2007; 1:53-57.

2. Wu HC, Wang CC, Fu YC.Surgical Management for Complications during Closure of Atrial Septal Defect with Amplatzer Device. Acta Cardiol Sin 2013; 29:557-61.

3. Yetkin U, Yurekli I, Akyildiz ZI. Emergency surgical approach to device emboli due to migration of the atrial septal defect occlude. Arch Med Sci 2014; 10: 464-69. 
4. Raghuram AR, Krishnan R, Kumar S. Complications in atrial septal defect device closure. Interactive CardioVascular and Thoracic Surgery 2008; 7:167-69.

5. Contrafouris C A, Chatzis AC, Giannopoulos NM. Emergency Surgery for Embolization of Amplatzer device into the Left Atrium. Hellenic J Cardiol 2006; 47: 245-46.

6. Seren M, Çiçek OF, Düzgün AC. Surgical treatment of two different interventional cardiological complications at the same patient. Cardiovascular Surgery and Interventions 2014;1:6-8.

Fig. I Echo showing the dislodged device into left atrium

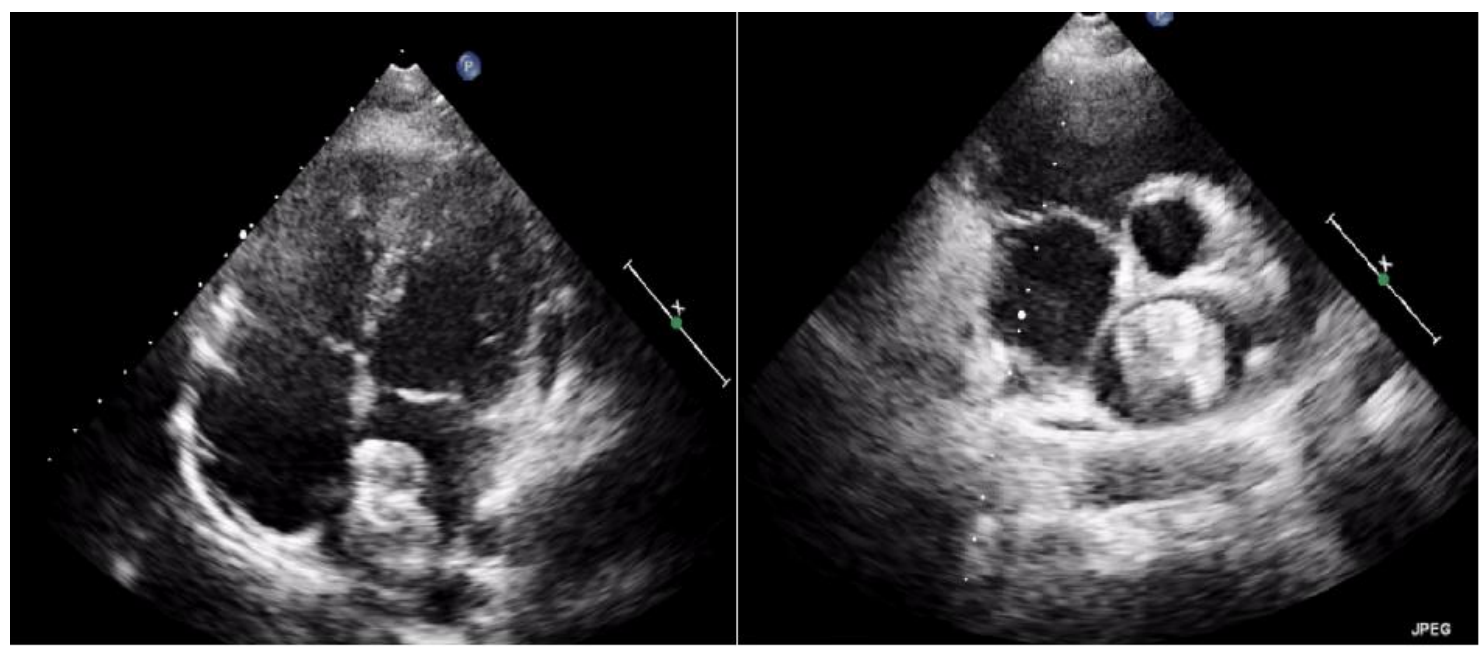

Fig. II Postoperative echo showing closure of ASD defect

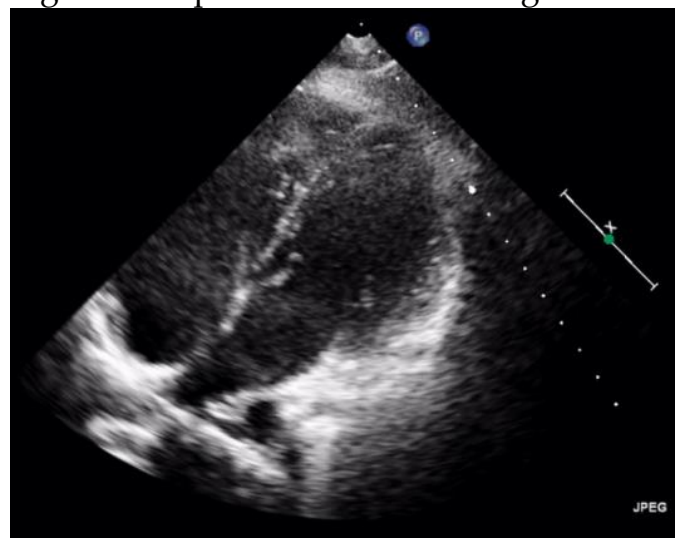

Fig. III Intra operative picture showing 1- ASD device through the defect, 2- Right atrium

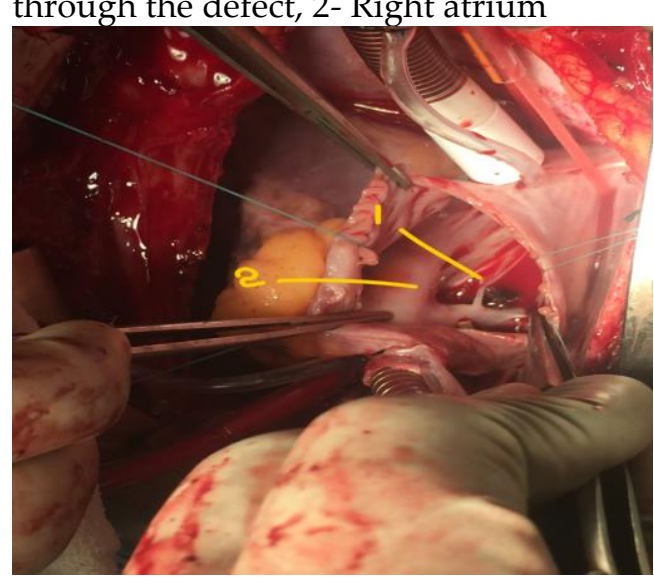

Fig. IV Retrieved Occluder device with fresh clots

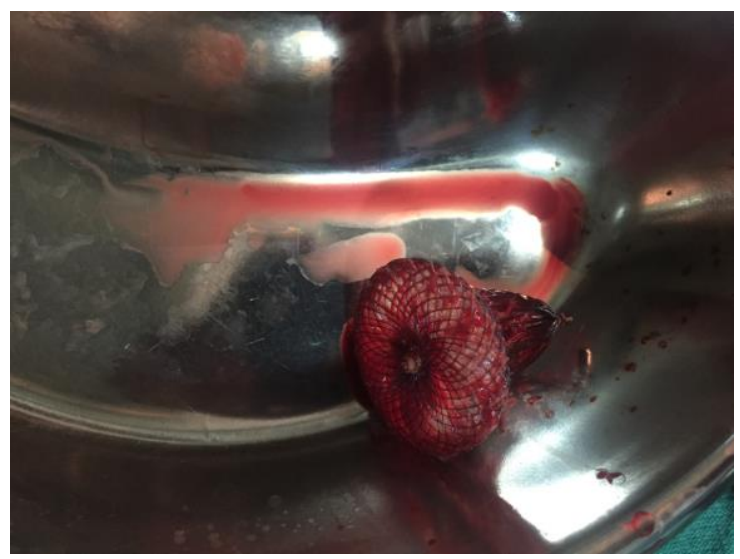

